\title{
Assessment of depth of anesthesia using principal component analysis
}

\author{
Mina Taheri ${ }^{1}$, Behzad Ahmadi ${ }^{2}$, Rassoul Amirfattahi ${ }^{3}$ and Mojtaba Mansouri ${ }^{4}$ \\ ${ }^{1,2,3}$ Digital Signal Processing Research Lab, Department of Electrical and Computer Engineering, Isfahan University of Technology, Isfahan, 84156-83111, Iran \\ 1,2,3,4 Medical Image and Signal Processing Research Center, Isfahan University of Medical Sciences, Isfahan, Iran. Correspondence should be addressed to Mina \\ Taheri (mh.taheri@ec.iut.ac.ir)
}

Received June $9^{\text {th }}, 2008$; revised October $12^{\text {th }}, 2008$; accepted November $13^{\text {th }}, 2008$

\begin{abstract}
A new approach to estimating level of unconsciousness based on Principal Component Analysis (PCA) is proposed. The Electroencephalogram (EEG) data was captured in both Intensive Care Unit (ICU) and operating room, using different anesthetic drugs. Assuming the central nervous system as a 20-tuple source, window length of 20 seconds is applied to EEG. The mentioned window is considered as 20 nonoverlapping mixed-signals (epoch). PCA algorithm is applied to these epochs, and largest remaining eigenvalue (LRE) and smallest remaining eigenvalue (SRE) were extracted. Correlation between extracted parameters (LRE and SRE) and depth of anesthesia (DOA) was measured using Prediction probability $\left(P_{K}\right)$. The results show the superiority of SRE than LRE in predicting DOA in the case of ICU and isoflurane, and the slight superiority of LRE than SRE in propofol induction. Finally, a mixture model containing both LRE and SRE could predict DOA as well as Relative Beta Ratio (RBR), which expresses the high capability of the proposed PCA based method in estimating DOA.
\end{abstract}

Keywords: Bispectral Index, Depth of Anesthesia, Eignevalue Decomposition, Principal Component Analysis

\section{INTRODUCTION}

To provide optimal working conditions for surgeons in the operating room as well as ensuring patient's safety, an anesthesiologist's effort is absolutely essential. However, patient awareness during surgery with the rate of $1: 1000$ [1] and over dosing with anesthetic agents is of major clinical concerns of anesthesia. Therefore, the necessity to assess and monitor the depth of anesthesia (DOA) is obvious. In conventional methods, DOA is measured based on the monitoring of several physiological signals such as respiration pattern, blood pressure, body temperature, tearing, sweating and heart rate [1], even though these signals are affected indirectly by anesthetic agents. On the other hand, these agents have significant effects on the electroencephalogram (EEG) waveform.

A large amount of information can be extracted from EEG waveform based on different signal processing methods. Ability of this information to predict DOA depends on the variation of its value in different levels of anesthesia. In general, the goal is to produce a unit-less EEG-based index that monotonically quantifies DOA. Several methods are available that have recently been reviewed by Freye et al. [2] and Jameson et al. [3].

The earliest methods were based on the FFT analysis of EEG signals. These approaches tend to find parameters that describe spectrum characteristics. Peak power frequency (PPF), median power frequency (MPF), and spectral edge frequency (SEF) have been the first descriptors in this field. Another parameter extracted from spectrum was the ratio of power in two empirically derived frequency bands [4]. In a work presented by Traast et al. [5] the power of EEG in different frequency bands was determined and the results indicate pronounced changes in EEG during emergence from propofol/sufentanil total intravenous anesthesia.

Zikov et al [6] proposed a wavelet based anesthetic value for central nervous system monitoring ( $\left.\mathrm{WAV}_{\mathrm{CNS}}\right)$ that quantifies the depth of consciousness between awake and isoelectric state. Their proposed technique is based on the analysis of the single-channel (frontal) EEG signal using stationary wavelet transform (SWT). The wavelet coefficients calculated from the EEG are pooled into a statistical representation which is then compared to well-defined awake and isoelectric states. Presenting a clinical study, they compared this technique with BIS monitor (Aspect Medical Systems, MA) as a reference and showed that they are well correlated $(\mathrm{r}=0.969)$. Furthermore, $\mathrm{WAV}_{\mathrm{CNS}}$ had a faster algorithm than BIS and was well suited for use as a feedback sensor in advisory systems and closed-loop control schemes.

Ferenets et al [7] analyzed the performance of several new measures based on the regularity and complexity of the EEG signal. These measures consist of spectral en- 
tropy (SpEn), approximate entropy (ApEn), and Higuchi fractal dimension (HFD) and Lempel-Ziv complexity (LZC). Their results show superior ability of the mentioned measures to predict DOA. Due to the arguments presented in their paper it is not feasible to point out "the best" EEG measure for the assessment of the depth of sedation, their results indicate that the measures sensitive to both the power spectrum as well as the amplitude distribution, i.e., the ApEn, LZC and HFD, perform slightly better than the other two tested measures. In the case of their tested measures, they recommend window length of $20 \mathrm{~s}$.

Application of neural networks (NN) in estimating DOA is reviewed by Robert et al [8]. They examined a large number of EEG derived parameters as NN inputs including spectral, entropy, complexity, bicoherence, wavelet transformation derived, autoregressive modeling and hemodynamic parameters as well as a great NN topology such as MLP and Self-Organizing networks. Finally, they recommended a two hidden layers MLP model or an ART model in which their weights are continuously updated after training phase. Moreover the use of qualitative parameters, besides quantitative ones, as network inputs is recommended. In a recent work by Lalitha et al [9] non-linear chaotic features and neural network classifiers are used to detect anesthetic depth levels. Chaotic features consist of correlation dimension (CD), Lyapunov exponent (LE) and Hurst exponent (HE) are used as features and two neural network models, i.e., multi-layer perceptron network (feed forward model) and Elman network (feedback model) are used for classification. Their experimental results show that the Lyapunov exponent feature with Elman network yields an overall accuracy of $99 \%$ in detecting the anesthetic depth levels.

According to various mentioned methods, different EEG monitors have been developed. The Narcotrend ${ }^{\mathrm{TM}}$ monitor (Monitor Technik, Bad Bramsted, Germany) that is based on pattern recognition of the raw EEG and classifies the EEG into different stages, introduces a dimensionless Narcotrend ${ }^{\mathrm{TM}}$ index from 100 (awake) to 0 (electrical silence). The algorithm uses parameters such as amplitude measures, autoregressive modeling, fast Fourier transform (FFT) and spectral parameters [10]. The SEDLine ${ }^{\mathrm{TM}}$ EEG monitor capable of calculating of PSI $^{\mathrm{TM}}$ index uses the shift in power between the frontal and occipital areas. The mathematical analysis includes EEG power, frequency and coherence between bilateral brain regions [11]. Datex-Ohmeda ${ }^{\mathrm{TM}} \mathrm{S} / 5$ entropy Module uses entropy of EEG waves to predict DOA [3] and finally BIS ${ }^{\mathrm{TM}}$ (Aspect Medical Systems, Newton, MA), that is the first monitor in the marketplace and has become the benchmark comparator for all other monitors, introduces the BISTM index (that is a unit-less number between 100 and 0 ) as a DOA indicator based on combination of spectral, bispectral and temporal analysis [4]. Approximately 450 peer-reviewed publications between 1990 and 2006 have examined the effectiveness, accuracy and usefulness, both clinical and economical, of the BIS $^{\text {TM }}$ monitor [3].
The aim of Principal Component Analysis (PCA) is to find source signals which are gaussian and uncorrelated. PCA can be interpreted in terms of blind source separation methods inasmuch as PCA is like a version of ICA in which the source signals are assumed to be gaussian. In other words, PCA finds a matrix which transforms the signal mixtures into a new set of uncorrelated signals. Extracted signals are ordered via PCA according to their variances (variance can be equated with power or amplitude). Consequently, the function of PCA is more than simply finding a transformation of the signal mixtures [12].

PCA has been widely used in pattern recognition and signal processing. The major applications and examples are engineering and scientific disciplines, e.g., in data compression, feature extraction, noise filtering, signal restoration, and classification [13]. PCA is used widely in data mining as a data reduction technique. In image processing and computer vision, PCA representations have been used for solving problems such as face and object recognition, tracking, detection, background modeling, parameterizing shape, appearance, and motion [14, 15]. In [16], the noise sensitivity, specificity and accuracy of the PCA method is evaluated by examining the effect of noise, base-line wander and their combinations on the characteristics of ECG for classification of true and false peaks.

The most important biomedical application of ICA and PCA is identifying different types of generators of the EEG as well as identifying its magnetic counterpart (MEG) [17]. MEG measurements give basically very similar information to EEG, but with a higher spatial resolution. MEG is mainly used for basic cognitive brain research. Another contribution is noise cancellation for brain signals such as electroencephalograms and magnetoencephalograms (EEG/MEG). References [18,19] introduced a new method to separate brain activity from artifacts using ICA. The approach is based on the assumption that the brain activity and the artifacts, e.g. eye movements or blinks, or sensor malfunctions, are anatomically and physiologically separate processes, and this separation is reflected in the statistical independence between the magnetic signals generated by those processes. In addition, ICA has been applied to problems in fields as diverse as speech processing, brain imaging (e.g., fMRI and optical imaging [20]), electrical brain signals (e.g., EEG signals), to extract features from a special array of electroencephalographic electrodes. The ICA framework can also be used for feature extraction from other kinds of data, for example, color and stereo images [21,22].

Additionally, EEG from patients undergoing surgery was collected. We introduce a novel method based on PCA. Our concentration would be on eigenvalues and eigenvectors. Finally, based on proper statistical methods and our data bank, the correlation between the extracted parameters and BIS index is observed. The reminder of paper is organized as follows: In part 2, methods and materials is described. The results and discussion are presented in section 3 , and section 4 contains the final conclusion. 


\section{METHODS AND MATERIALS}

In this section, the experiment, the data acquisition, and the data analysis are described.

\subsection{Patients}

Following the approval of the ethical committee of the medical school, eight coronary artery bypass graft surgery candidates were selected ( 6 males, 2 females, of average age 56.2 years and the average weight of $68.3 \mathrm{~kg}$ ) and written informed consents were obtained from all selected subjects. Inclusion criteria were absent of neurological disorders such as cerebrovascular accidents and convulsions. Preoperative neurological complications (such as cerebral emboli and convulsion) caused exclusion from the study. The anesthesiologist performed Preoperative evaluation on the day before surgery. For anxiolysis, the patients were premedicated by intramuscular morphine $0.1 \mathrm{mg} / \mathrm{kg}$ and promethazine $0.5 \mathrm{mg} / \mathrm{kg}$, 30 minutes before transfer to operating room. After arrival in operating room, electrocardiogram, pulse oxymetry, depth of anesthesia, and invasive blood pressure monitoring was established. The BIS-QUATTRO sensorTM (Aspect Medical Systems, Newton, MA) applied to the forehead of the patients before induction of anesthesia. Then 8 patients after preoxygenation with $\mathrm{O} 2$, were anesthetized in the same manner by intravenous thiopental sodium $(5 \mathrm{mg} / \mathrm{kg})$, pancuronium bromide $(0.1$ $\mathrm{mg} / \mathrm{kg})$, fentanyl $(5 \mu \mathrm{g} / \mathrm{kg})$, and lidocaine $(1.5 \mathrm{mg} / \mathrm{kg})$. After the induction of anesthesia and until cardiopulmonary bypass beginning, anesthesia continued by administration of isoflurane (1 MAC), morphine $(0.2$ $\mathrm{mg} / \mathrm{kg}$ ) and $\mathrm{O} 2(100 \%)$. During coronary artery bypass grafting under $\mathrm{CPB}$, patients were anesthetized by propofol $(50-150 \mu \mathrm{g} / \mathrm{kg} / \mathrm{min})$ under BIS control (40-60) and $\mathrm{O} 2(80 \%)$. For organ protection during $\mathrm{CPB}$, patients were undergone mild hypothermia $\left(31-33^{\circ} \mathrm{C}\right)$. After coronary artery bypass grafting and patients rewarming and obtaining standard CPB separation criteria, the patients gradually were weaned from CPB. After separation from $\mathrm{CPB}$, anesthesia was continued by isoflurane (1 $\mathrm{MAC})$ and $\mathrm{O} 2(100 \%)$ administration to the end of surgery. After surgery, patients were transported to ICU under portable monitoring and manual ventilation. In the ICU mechanical ventilation with $60 \%$ fractioned inspired oxygen and standard homodynamic monitoring were continued. In ICU and until complete recovery, the sedative regimen was intravenous morphine $(2 \mathrm{mg})$ if needed. In this study the raw EEG data and relative BIS index were collected during whole period of operation from operative room arrival to complete recovery in the intensive care unit.

\subsection{Data Acquisition}

The EEG signal was collected by using a BIS-QUATTRO Sensor ${ }^{\mathrm{TM}}$ that was composed of self- adhering flexible bands holding four electrodes, applied to the forehead with a frontal-temporal montage.

The used EEG lead was Fpz-At1, and the reference lead was placed at FP1. The sensor was connected to a
BIS-X-P Monitor and all binary data packets containing raw EEG data wave signals and BIS index which is converted to binary format using an A/D converter operating with $128 \mathrm{~Hz}$ sampling frequency were recorded via an RS232 interface on a laptop using a Bi-spectrum analyzer developed with $\mathrm{C}++$ Builder by Satoshi Hagihira [23]. The algorithms that are presented in this study were tested on these raw EEG signals.

The sensor was attached to the patient's forehead at the beginning of anesthesia and the data were collected continuously until he/she awoke at ICU. Therefore, in this study a large amount of EEG data with their BIS index was collected for each patient. Although DOA is an index beyond BIS index and BIS index needs to be validated and processed, in this paper the BIS index is considered as DOA for simplicity. Some other events such as changes of anesthesia regimen, intubations and applying $\mathrm{CPB}$ and transferring to ICU were recorded. Because of short acting time of thiopental sodium (approximately 15-20 sec), this part of EEG data was not analyzed.

\subsection{Principal Component Analysis}

PCA is a well-known technique in multivariate analysis and data mining. One of the properties of PCA is Eigenvalue Decomposition. The aim of PCA is to derive a relatively small number of decorrelated linear combinations (principal components) of a set of random zeromean variables while also retaining the signal information as much as possible [24].

Principal Components Analysis has the applications of dimensionality reduction, determination of linear combinations of variables, feature selection, multidimensional data visualization, and identification of underlying variables.

Often components with the smallest variances called minor components (MCs) are regarded as unimportant or associated with noise, while those within which the input data have the largest variances are regarded as important. However, in some applications, the MCs are of the same importance as the PCs, which is noteworthy here. In the proposed algorithm the MCs reveal meaningful information. In the case of feature extraction and dimension reduction, PCA proposes a method based on the eigen structure of data covariance matrix. If signals are zero-mean, the covariance and correlation matrices are identical. Applying the PCA or equivalently KarhunenLoeve transform (KLT) as a technique for eigenvectors and eigenvalues computation, the algorithm could be formulated as follows. Let $X$ the signal to be analyzed, then

$$
\hat{R}_{X X}=E\left\{X(K) X^{T}(K)\right\}=V \Lambda V^{T} \in \mathfrak{R}^{m \times m}
$$

Where $\hat{R}_{X X}=E\left\{X X^{T}\right\}$ is the covariance matrix of zero-mean signal $X$ and $E$ is the expectation operator. Also, $\Lambda=\operatorname{diag}\left\{\lambda_{1}, \lambda_{2}, \ldots, \lambda_{m}\right\}$ is a diagonal matrix containing $m$ eigenvalues and $V=\left[v_{1}, v_{2}, \ldots v_{m}\right] \in \mathfrak{R}^{m \times m}$ are principal eigenvectors. Applying KLT as a linear transformation, principal and minor components could be 
extracted as follows

$$
y_{P}=V_{S}^{T} X
$$

Where $X=\left[x_{1}(k), x_{2}(k), \ldots, x_{m}(k)\right]^{T}$ is the zero-mean input vector and $y_{P}=\left[y_{1}(k), y_{2}(k), \ldots, y_{n}(k)\right]^{T}$ is the output vector called the vector of principal components (PCs) and $V_{S}=\left[v_{1}, v_{2}, \ldots, v_{n}\right]^{T} \in \mathfrak{R}^{m \times n}$ is the set of signal subspace eigenvectors, with the orthonormal vectors $v_{i}=\left[v_{i 1}, v_{i 2}, \ldots, v_{i m}\right]^{T}$. The vectors $v_{i}$ are eigenvectors of the covariance matrix, while the variances of the PCs $y_{i}$ are the corresponding principal eigenvalues. Minor components are

$$
y_{M}=V_{N}^{T} X
$$

Where $V_{N}=\left[v_{m}, v_{m-1}, \ldots, v_{m-n+1}\right]$ consists eigenvectors associated with the smallest eigenvalues [21]. The basic problem is the standard eigenvalue problem which can be formulated by the equations

$$
R_{X X} v_{i}=\lambda_{i} v_{i}, \quad i=1,2, \ldots, n
$$

Where $v_{i}$ are the eigenvectors and $\lambda_{i}$ are the corresponding eigenvalues. Note that the above equation can be written in matrix form $V^{T} R_{X X} V=\Lambda$.

In the standard numerical approach for extracting the principal components, first the covariance matrix $R_{X X}=E\left\{X X^{T}\right\}$ is computed and then its eigenvectors and corresponding eigenvalues are extracted by one of the known numerical algorithms. However, if the input data vectors have a large dimension, then the covariance matrix $R_{X X}$ becomes very large and it may be difficult to compute the required eigenvectors [24].

A neural network approach with adaptive learning algorithms enables us to find the eigenvectors and the associated eigenvalues directly from the input vectors without a need to compute or estimate the very large covariance matrix $\mathfrak{R}_{X X}$. Such an approach will be especially useful for nonstationary input data, i.e., in cases of tracking slow changes of correlations in the signals or in updating eigenvectors with new samples.

Every neuron inside the human brain acts like a small electric generator when it is active. If large numbers of neurons become simultaneously active it is possible to measure the resultant electrical effects at the scalp using an array of electrodes. Our virtual assumption is to simulate the central nervous system (CNS) as a 20-tuple source, which generate 20 signals. The EEG sensor attached to patient forehead collect different 20-tuple mixtures of these sources. Our aim is to track small changes, but due to time-domain nature of our analysis, small window lengths are more preferable. Fortunately, this would reduce the dimension. Nevertheless, large data vectors made the covariance matrix $R_{X X}$ very large. Different window and epoch (each mixed signal is named as an epoch) lengths have been investigated and at the end, window length of 20 seconds was selected for further analysis. After that, each window is divided into 20 equal and nonoverlapping epochs. So, epoch length is equal to one second. The mentioned epochs are considered as 20 mixed signals. Then, PCA analysis and especially eigenvalue decomposition are applied to the electroencephalogram (EEG). Thus, the covariance matrix $R_{X X}=E\left\{X X^{T}\right\}$ is computed and then its eigenvectors and associated eigenvalues are determined. The extracted eigenvalues presented an acceptable behavior in different depths of anesthesia. Our concentration was put specifically on largest remaining eigenvalue (LRE) and smallest remaining eigenvalue (SRE). The correlation between DOA and LRE were measured with regression analysis. The same was done for SRE and DOA.

\subsection{Statistical Analysis}

The coefficient of determination $\left(R^{2}\right)$ was calculated to evaluate the performance of different parameters and their combinations to predict DOA. Statistical significance was assumed at probability levels of $P \leq 0.05$. Our aim was to maximize the correlation between the measured sub- parameters (LRE and SRE) and BIS index, i.e., it is equivalent to nonlinear regression with ordinary least squares. Also, the correlation between BIS index and the extracted sub-parameters was investigated with the model-independent Prediction Probability (Pk) [25]. As a nonparametric measure, the $\mathrm{Pk}$ is independent of scale units and does not require knowledge of underlying distributions or effort to linearize or otherwise transform scales. A Pk value of 1 means that the predicting variables (LRE and SRE) always predict the value of the predicted variable (e.g., BIS index) correctly. Pk value of 0.5 means that predictors predict no better than only by chance. The $\mathrm{Pk}$ values were calculated on a spreadsheet using the Excel 2003 software program and the PKMACRO written by Warren Smith [25]. In the case of inverse proportionality between indicator and indicated parameters, the actual measured $\mathrm{Pk}$ value is $1-\mathrm{Pk}$.

Another statistical analysis used in this study was ordinal logistic regression. This regression examines the relationship between one or more predictors and an ordinal response. The index that determines the efficiency of this regression model is called "Concordant", which shows the percentage of values predicted successfully with the model.

\section{RESULTS AND DISCUSSION}

The results were classified in drug groups (isoflurane and propofol) and Intensive Care Unit (ICU). The correlation between the extracted parameters and BIS index (Bispectral index) is measured by means of the statistical methods described in the previous section and the results are presented.

\subsection{SRE}

First and foremost, it can be concluded that SRE is directly proportional to the BIS index. The scatterplots depicted in Figure 1 and Figure 2 show the above assert clearly.

Figure 3 compares the efficiency of SRE and LRE in predicting depth of anesthesia in different groups (ICU and drugs). In this figure, concordant was used as a statistical measure. 


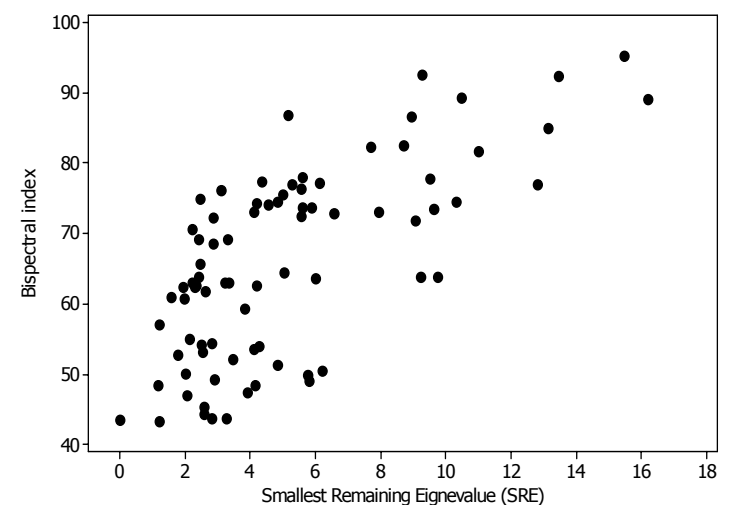

Figure 1. BIS index versus Smallest Remaining Eigenvalue (SRE) in ICU

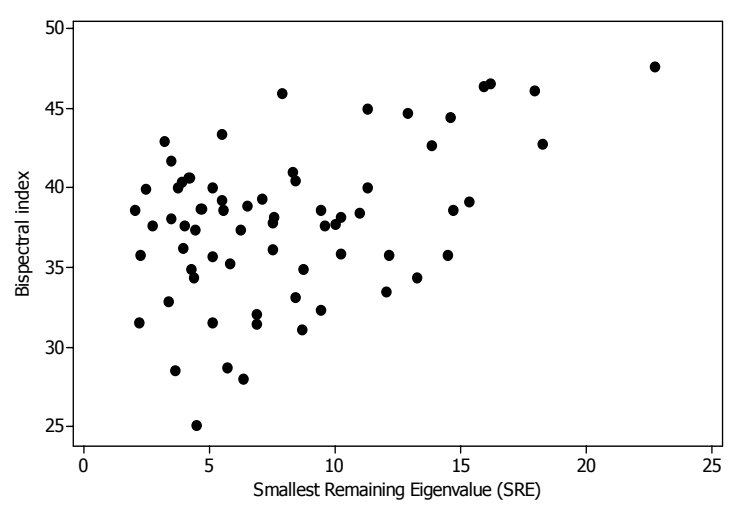

Figure 2. BIS index versus Smallest Remaining Eigenvalue (SRE) in propofol

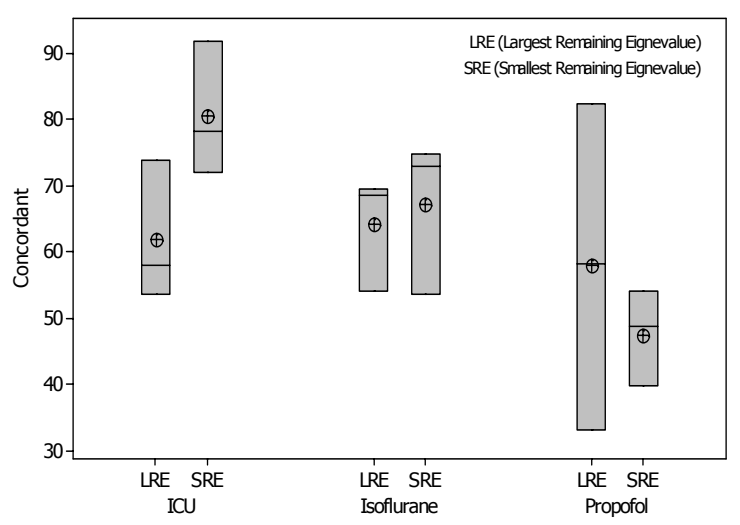

Figure 3. Concordant for different groups versus SRE and LRE

The results show the higher capability of SRE when it is used as a measure of DOA in ICU, rather than when it is used as measure of DOA in isoflurane and propofol groups. For further analysis, prediction probability (PK) was used. PK values are presented as mean \pm STD in Table 1 .

It should be noted that the $\mathrm{P}_{\mathrm{k}}$ values are calculated for the whole BIS index range and without being divided into predetermined groups. This is the reason of the existence of smaller values in comparison with concordant values. The values in Table 1 corroborate the results of Figure 3.
Table 1. Prediction probabilities of different group for SRE

\begin{tabular}{cc}
\hline Different Groups & Prediction ProBABILITY \\
\hline ICU & $65.5 \pm 8 \%$ \\
Isoflurane & $63 \pm 8 \%$ \\
Propofol & $58.5 \pm 10 \%$ \\
\hline
\end{tabular}

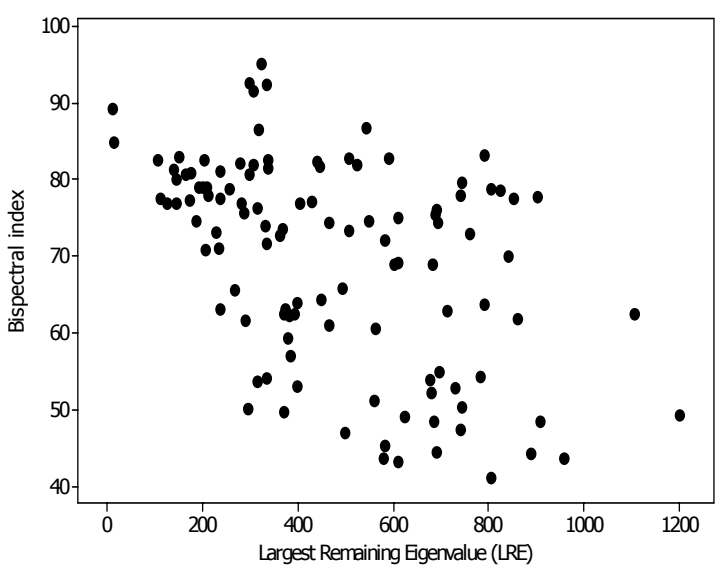

Figure 4. BIS index versus Largest Remaining Eigenvalue $(\mathrm{LRE})$ in ICU

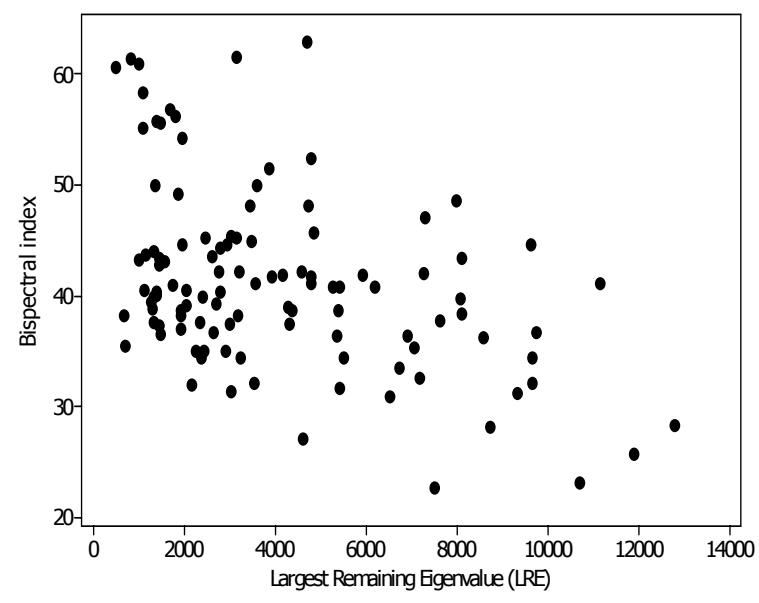

Figure 5. BIS index versus Largest Remaining Eigenvalue (LRE) in isoflurane

Table 2. Prediction probabilities of different group for LRE

\begin{tabular}{lc}
\hline Different Groups & Prediction Probability \\
\hline ICU & $60 \pm 6.97 \%$ \\
Isoflurane & $60.5 \pm 8 \%$ \\
Propofol & $62.5 \pm 9 \%$ \\
\hline
\end{tabular}

\subsection{LRE}

LRE is inversely proportional to the BIS index, that is, LRE increases with the increasing depth of anesthesia. Scatterplots shown in Figure $\mathbf{4}$ and Figure $\mathbf{5}$ could prove the above claim.

In order to compare the efficiency of LRE algorithm in different groups we should refer to Figure 3. This figure indicates that there is no obvious superiority in any of these groups. 
In this case prediction probability $\left(\mathrm{P}_{\mathrm{K}}\right)$ provides us with a more precise insight. $\mathrm{P}_{\mathrm{K}}$ values are presented as mean \pm STD in Table 2. The measured $\mathrm{P}_{\mathrm{K}}$ values presented in Table 2 confirm the result of Figure 3 for LRE. The only extra information extracted from Table 2 is that in the case of propofol induction the results are slightly better.

Finally, Figure 3 expresses the superiority of SRE than LRE in predicting DOA in the case of ICU and isoflurane induction, and the slight superiority of LRE than SRE in propofol induction.

\subsection{Relative Beta Ratio}

Finally a mixture model including both LRE and SRE is compared to the model containing Relative Beta Ratio (RBR). RBR is calculated as

$$
R B R=\log \frac{P_{30-47 \mathrm{~Hz}}}{P_{11-20 \mathrm{~Hz}}}
$$

Where, $P_{30-47 \mathrm{~Hz}}$ and $P_{11-20 \mathrm{~Hz}}$ indicate the power spectral density in frequency ranges of $30-47 \mathrm{~Hz}$ and $11-20 \mathrm{~Hz}$, respectively. A mixture model is a model in which all of the model parameters are involved in predicting the desired index. For instance, in our mixture model, BIS index is predicted using both LRE and SRE parameters. RBR is said to be the main parameter in calculating the BIS index and is referred to as an effective and critical parameter in predicting depth of anaesthesia [4]. Figure 6 reveals a high similarity between the proposed mixture model containing both LRE and SRE parameter and the main parameter of BIS monitor which is called RBR.

In this figure, the group containing isoflurane induction is omitted. Both of the parameters (LRE and SRE) perform more satisfactorily in ICU than propofol induction.

\section{CONCLUSION}

A method based on PCA is proposed to estimating DOA. The principal components are extracted and the related eigenvalues are calculated as well. The smallest and largest eigenvalues express a meaningful behavior due to the changes of BIS index. So, the above parameters are selected for estimating DOA.

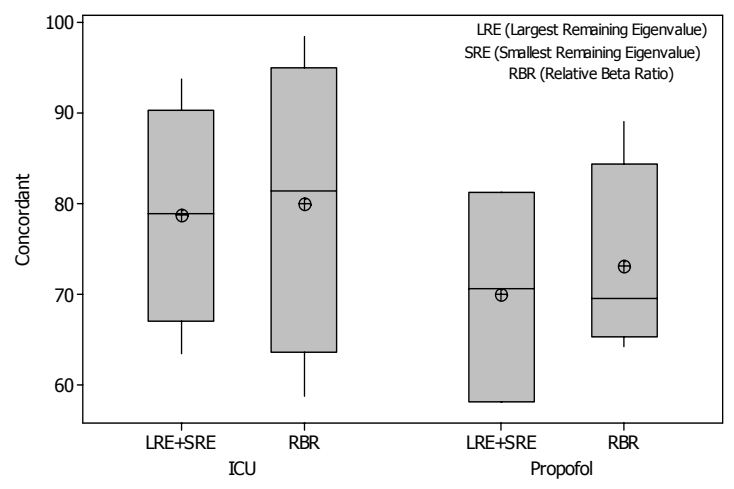

Figure 6. Comparison of RBR and proposal mixture model based on concordant values
The main result of applying the foregoing algorithm is that SRE is directly proportional to the BIS index. On the other hand, LRE is inversely proportional to the BIS index. All in all, the results show an acceptable correlation between the extracted parameters and DOA. The LRE and SRE are extremely capable of estimating DOA, especially in ICU. This is due to the ability of PCA in calculating the changes in signal energy and the changes in signals complexity as well. On the other hand, it is shown [7] that the EEG signal complexity changes meanwhile patients level of consciousness vary. Thus, PCA could be a powerful tool for predicting BIS index.

Except in propofol, the SRE parameter could predict the BIS index better than LRE. Consequently, the mixture model containing both LRE and SRE is approximately equal to a model containing RBR in predicting BIS index.

Another point that should be mentioned is that the original BIS is in fact much more than its components. The elaborate artifact rejection algorithms as well as the nature of the nonlinear function to combine the components have an important impact on the original BIS value, which were not considered in this study. Consequently, in order to improve the accuracy of the depth of anesthesia estimation, comparison against sedation scales (such as $\mathrm{OAA} / \mathrm{S}$ ) and drug levels is needed. The reason is that BIS is not equal to depth of anesthesia but needs to be validated for DOA assessment itself.

The work reported is preliminary. Although the results are significant, wide patient population is necessary for better evaluation. In conclusion, the approach used in this work based on the application of PCA could propose the use of PCA in estimating DOA.

\section{ACKNOWLEDGMENT}

The authors would like to thank Dr. Satoshi Hagihira due to his notification and problem solving in the application of Bispectrum Analyzer and Dr. Warren Smith that made PKMACRO available.

\section{REFERENCES}

[1] R. D. Miller, (2005) Miller's Anesthesia, Sixth edition, Elsevier Churchill Livingstone, 1227-1264.

[2] E. Freye and J. V. Levy, (2005) "Cerebral monitoring in the operating room and the intensive care unit: An introductory for the clinician and a guide for the novice wanting to open a window to the brain. Part I: The electroencephalogram", J Clin Monit Comput, 1-76.

[3] L. C. Jameson and T.B. Sloan, (2006) "Using EEG to monitor anesthesia drug effects during surgery", J Clin Monit Comput, 445-472.

[4] I. J. Rampil, (1998) "A primer for EEG signal processing in anesthesia”, Anesthesiology, 980-1002.

[5] H. S. Traast and C. J. Kalkman, (1995) "Electroencephalographic Characteristics of emergence from propofol/sufentanil total intravenous anesthesia", Anesth Analg, 366-371.

[6] T. Zikov, S. Bibian, G. A. Dumont, M. Huzmezan, and C. R. Ries, (2006) "Quantifying cortical activity during general anesthesia using wavelet analysis", IEEE Trans. Biomed. Eng., Vol. 53, No. 4, 617-632.

[7] R. Ferenets, T. Lipping, A. Anier, V. Jäntti, S. Melto, and S. Hovilehto, (2006) "Comparison of entropy and complexity meas- 
ures for the assessment of depth of sedation", IEEE Trans. Biomed. Eng., Vol. 53, No. 6, 1067-1077.

[8] C. Robert, P. Karasinski, C. D. Arreto, and J. F. Gaudy, (2002) "Monitoring Anesthesia using neural networks: A survey", J Clin. Monit. Comput, Vol. 17, 259-267.

[9] V. Lalitha and C. Eswaran, (2007) "Automated Detection of Anesthetic Depth Levels Using Chaotic Features with Artificial Neural Networks", J Med Syst, 445-452.

[10] R. Bender, B. Schultz, and U. Grouven, (1992) "Classification of EEG signals into general stages of anesthesia in real time using autoregressive models", Conf Proc of the 16th Annual Conference of the Gesellschaft fur Klassifikatione, University of Dortmund.

[11] D. R. Drover, H. J. Lemmens, E. T. Pierce, et al, (2002) "Patient State Index: titration of delivery and recovery from propofol, alfentanil, and nitrous oxide anesthesia", Anesthesiology, 82-89.

[12] J. V. Stone, (2004) Independent Component Analysis: A Tutorial Introduction, Bradford Book.

[13] K. I. Diamantaras and S. Y. Kung, (1996) Principal Component Neural Networks. Theory and Applications, Adaptive and Learning Systems for Signal Processing, Communications and Control, John Wiley \& Sons Inc., New York.

[14] M. Turk and A. Pentland, (1991) "Eigenfaces for recognition", Journal of Cognitive Neuroscience, 71-86.

[15] S. Y. Kung, K. Diamantaras, and J. Taur. (1991) "Neural networks for extracting pure/constrained/oriented principal components. In J. R. Vaccaro, editor", SVD and Signal Processing El-sevier Science, Amsterdam, 57-81.
[16] M. P. S. Chawla, (2008) "A comparative analysis of principal component and independent component techniques for electrocardiograms", Neural Computing \& Applications.

[17] Hyv"arinen, A., J. Karhunen, and E. Oja, (2001) Independent Component Analysis., John Wiley \& Sons Inc., New York.

[18] R. Vigário, V. Jousmäki, M. Hämäläinen, R. Hari, and E. Oja, (1998) "Independent component analysis for identification of artifacts in magnetoencephalographic recordings", In Advances in Neural Information Processing Systems, MIT Press, Vol. 10, 229-235.

[19] S. Makeig, A. J. Bell, T. P. Jung, and T. Sejnowski, (1996) "Independent component analysis of electroencephalographic data", Advances in Neural Information Processing Systems, MIT Press, Vol. 8, 145-151.

[20] G. D. Brown, S. Yamada, and T. J Sejnowski, (2001) "Independent components analysis (ica) at the neural cocktail party", Trends in neuroscience, Vol. 24, 54-63.

[21] P. O. Hoyer and A. Hyv"arinen, (2000) "Independent component analysis applied to feature extraction from colour and stereo images", Network: Computation in Neural Systems, Vol. 11, 191-210.

[22] Parra, L., C. D. Spence, P. Sajda, A. Ziehe, and K.-R. M“uller, (2000) Unmixing hyperspectral data. In Advances in Neural Information Processing Systems 12, MIT Press, 942-948.

[23] S. Hagihira, M. Takashina, T. Mori, T. Mashimo, and I. Yoshiya, (2001) "Practical issues in bispectral analysis of electroencephalographic signals", Anesth Analg, Vol. 93, 966-970. 